\title{
Acute Exercise Effects on Measures of Attention and Impulsivity in Children with Attention Deficit/Hyperactivity Disorder
}

\author{
Anthony D. Mahon ${ }^{1}$, Raymond S. Dean ${ }^{2}$, David E. McIntosh ${ }^{3}$, Andrea D. Marjerrison ${ }^{1}$, Andrew S. Cole ${ }^{1}$, Megan \\ E. Woodruff ${ }^{1} \&$ Mary P. Lee ${ }^{1}$ \\ ${ }^{1}$ Human Performance Laboratory, Ball State University, Muncie, IN, USA \\ ${ }^{2}$ Neuropsychology Laboratory, Ball State University, Muncie, IN, USA \\ ${ }^{3}$ Department of Special Education, Ball State University, Muncie, IN, USA \\ Correspondence: Anthony D. Mahon, Ph.D. Human Performance Laboratory, Ball State University, Muncie, IN \\ 47306, USA. Tel: 1-765-285-8693. E-mail: tmahon@bsu.edu
}

Received: March 28, 2013

Accepted: July 22, 2013

Online Published: September 23, 2013

doi:10.5539/jedp.v3n2p65

URL: http://dx.doi.org/10.5539/jedp.v3n2p65

\begin{abstract}
This study examined the effect of a single bout of exercise on measures of attention and impulsivity in children with attention deficit/hyperactivity disorder (ADHD). Children with ADHD ( $\mathrm{n}=21,11.3 \pm 1.8 \mathrm{yrs})$ and children without ADHD ( $\mathrm{n}=21,11.6 \pm 1.9 \mathrm{yrs})$ participated in the study. After performing an initial exercise test to measure peak aerobic exercise capacity, the children reported to the laboratory for 2 additional trials. For children with ADHD one trial was performed off medication and the other trial occurred on medication. During each testing session the Connor's Continuous Performance Test II (CCPT II) was performed immediately before and after 20 minutes of intermittent exercise (30 sec exercise/30 sec rest) at $90 \%$ of peak aerobic work rate. Errors of omission, errors of commission, and reaction time (t-scores) were assessed from the CCPT II. The data were analyzed with a 3-way (group $\mathrm{x}$ trial $\mathrm{x}$ time) MANOVA. There was a significant increase in the error of omission t-score over time (pre to post exercise). There were no significant findings for the error of commission $\mathrm{t}$-score. In the ADHD group the reaction time score was significantly higher than children without ADHD, significantly decreased with medication, and significantly increased over time. No other interaction or main effects were observed. Further research identifying the optimal exercise intensity and duration that can improve behavior, neurocognitive function, and academic performance is warranted.
\end{abstract}

Keywords: ADHD, boys, girls, physical activity

\section{Introduction}

\subsection{Statement of the Problem}

Attention deficit/hyperactivity disorder (ADHD) is a neuropsychological disorder that is manifested by poor attention and hyperactive and impulsive behaviors. These behaviors are very disruptive to children's social and cognitive development, and impair academic progress (Barkley, 1998; Biederman, 2005; Biederman \& Faraone, 2005; Greenhill, 1998). The most common form of treatment for ADHD is the prescription of stimulant medications such as methylphenidate and amphetamine (Bennett, Brown, Craver, \& Anderson, 1999). These medications, although not a cure for ADHD, improve many of the maladaptive behaviors associated with the disorder by presumably enhancing dopaminergic and noradrenergic activity in the central nervous system (Swanson \& Volkow, 2009; Volkow et al., 1998; Wilens, 2006). However, apprehensions regarding the side effects associated with stimulant medication use (Bennett, et al., 1999; Biederman \& Faraone, 2005; Wolraich, McGuinn, \& Doffing, 2007) raise concerns among some parents about this treatment option (Charach, Skyba, Cook, \& Antle, 2006).

Alternative or in combination with the use of stimulant medication are other forms of treatment. Specifically, counseling and behavior modification methods may serve to supplement stimulant medication use and prove effective in the overall management of ADHD (AAP, 2001; Benner-Davis \& Heaton, 2007; Wolraich, 1997). Another form of non-pharmacological treatment that may have promising benefits in children with ADHD, and has been the subject of both anecdotal (Bass, 1985; Higdon, 1999) and experimental reports (Berwid \& Halperin, 2012; Chang, Liu, Yu, \& Lee, 2012; Medina et al., 2010; Pontifex, Saliba, Raine, Picchietti, \& Hillman, 2013; 
Tantillo, Kesick, Hynd, \& Dishman, 2002; Verret, Guay, Berthiaume, Gardiner, \& Beliveau, 2012), is the effect of exercise on behavior and neurocognitive function. Indeed, although a wealth of empirical evidence is lacking, exercise may stimulate the brain in a manner that is similar to the effect of stimulant medication (Tantillo, et al., 2002).

\subsection{Purpose and Hypotheses}

Ascertaining the benefits of exercise in children with ADHD is necessary in order to determine the potential therapeutic use. Therefore, this study was designed to examine the effect of a single bout of vigorous aerobic exercise on performance on the Conner's Continuous Performance Test II (CCPT II (Connors \& Staff, 2002)) in boys and girls with and without ADHD. For children with ADHD, trials were conducted with and without the influence of medication. It was hypothesized that in the absence of medication, exercise would significantly improve CCPT II performance in children with ADHD so post-exercise performance on the CCPT II would be similar to the performance observed in children with ADHD on medication and in children without ADHD.

\section{Method}

\subsection{Participants}

Twenty-one children without ADHD (10 boys and 11 girls) and 22 children with ADHD (20 boys and 2 girls) and currently using stimulant medication were subjects in this study. All children were between 8 and 14 years of age and comprised the full range of pubertal stages (1 to 5) based on Tanner's indices of pubic hair development (Tanner, 1962) according to self/parental assessment. As established by parental report, children without ADHD had no known history of neuropsychological disorders or were using medication for such disorders. For children with ADHD a diagnosis consistent with Diagnostic and Statistical Manual for Mental Disorders-Fourth Edition, Text Revised procedures (APA, 2000) was confirmed in 20 of the children by the child's physician. Children with ADHD also were on a stable dose of stimulant medication for 6 months or longer and were free of any other known neuropsychological disorder and use of other medications according to parental report. Children were required to report to the laboratory on four separate days for testing. The cardiorespiratory and perceptual responses to the graded exercise test obtained from participants in this study were previously published (Mahon, Woodruff, Horn, Marjerrison, \& Cole, 2012).

\subsection{Instruments}

The Kaufman Brief Intelligence Test (K-BIT IQ) was designed to assess individuals between the ages of 4 to 90 years. The K-BIT IQ Composite score is composed of the Vocabulary and Matrices subtests and has a mean of 100 and a standard deviation of 15 . Test-retest reliability was reported as .92 for the K-BIT IQ Composite score, .86 for the Vocabulary subtest, and .83 for the Matrices subtest children from age 5 to 12 (Kaufman \& Kaufman, 1990). For children age 13 to 19, test-retest reliability was reported as .93 for the K-BIT IQ Composite score, .96 for the Vocabulary subtest, and .80 for the Matrices subtest. The K-BIT manual (Kaufman \& Kaufman, 2004) reported strong concurrent validity with Wechsler Intelligence Scale for Children-Revised (WISC-R) demonstrating a high correlation of 80 between the WISC-R Full Scale IQ and K-BIT IQ Composite.

The Behavior Assessment System for Children (BASC (Reynolds \& Kamphaus, 1998)) was developed to assess behavior and personality of individuals between the ages of $2 \frac{1}{2}$ and 18 . The BASC consists of the three different scales: Teacher, Parent, and Self-Report. For the present study, only the Parent Rating Scale (PRS) was utilized. The PRS is composed of several composites (Externalizing Problems, Internalizing Problems, and Adaptive Scales) and is completed by the parent. The Externalizing Problems composite is comprised of the Aggression, Hyperactivity, and Conduct Problems scales, while the Internalizing Problems composite is composed of the Anxiety, Depression, and Somatization scales. The Attention Problems scale does not contribute to either of the aforementioned composites. For this study, the Hyperactivity and Attention scales were considered to affirm the diagnosis of an attention disorder. For the Hyperactivity and Attention Problems scales, a t-score of 41-59 is Average, 60-69 is At-Risk, and 70 and above is Clinically Significant. Test-retest reliability for the Hyperactivity scale ranges are considered high with median values ranging from .77 to .88 across all age groups (Reynolds \& Kamphaus, 1998). In addition, median values for the Attention Problems scale ranged from .78 to .92 across all age groups. Correlations with the Child Behavior Checklist (CBCL (Achenbach, 1991)) demonstrated good concurrent validity. Correlations between the Externalizing Composite on the CBCL and the BASC Hyperactivity scale ranged from .61 to .75 , while the correlations between the CBCL Attentions Problems scale and the BASC Attention Problems scale ranged from .67 to .78 .

The CCPT II (Connors \& Staff, 2002) is a computerized measure of attention used to assess executive functioning, sustained attention, and impulsivity with individuals from age 6 through adult. In addition, the 
measures authors note that the CCPT II is sensitive to medication change and can be used to assess improvement or deterioration related to drug dosage. The CCPT II is often used as one of the measures when assessing individuals who are at-risk for attention disorders. Research also has demonstrated that poor performance on the CCPT II is highly correlated with the presence of ADHD (Hale et al., 2009). Test-retest reliability (.87) with individuals diagnosed with attention disorders is considered good (Connors \& Staff, 2002). The discriminant validity of the CCPT II has been well established where scores have been found to be significantly different for normal compared to individuals with attention problems (Connors \& Staff, 2002; Hale, et al., 2009).

\subsection{Procedures}

Upon the first visit to the laboratory, parental permission and child assent were obtained. Height and weight were measured and age was recorded. Parents completed the hyperactivity and attention subscales of the BASC. For children without ADHD a parent completed one assessment. For children with ADHD, a parent completed two assessments. One assessment was to gauge their child's present status and the other assessment was a retrospective view of the child's behavior at the time of diagnosis. The K-BIT also was administered to each child. A parent of each child with ADHD provided information regarding their child's medication type, daily dose, years since diagnosis, and years on medication. The child then performed a practice test version of the CCPT II to become familiar with the procedures utilized during visits three and four. After this a practice graded exercise test was performed in order to allow the child to become acquainted with the exercise test procedures that were used in the next visit. This test was performed on an electrically-braked cycle ergometer (Lode, Groningen, The Netherlands) and began at 25 Watts (W) for 2 minutes and increased by $25 \mathrm{~W}$ every 2 minutes until a near maximal effort was reached. After a short rest, each child practiced a few repetitions of the intermittent exercise protocol that was used during the third and fourth visits. Heart rate (Polar Monitor) and rating of perceived exertion (RPE) using the OMNI 0-10 scale (Robertson et al., 2000) were assessed during the practice test. Prior to the exercise bout a standardized set of instructions was read by each subject regarding the use of the RPE scale.

On the second visit the child's peak exercise capacity during cycle ergometry was assessed. A graded exercise test protocol was used and the test commenced at 20 Watts (W) and increased by $20 \mathrm{~W}$ every 2 minutes to approximately $100-140 \mathrm{~W}$; thereafter, the work rate increased by $10 \mathrm{~W}$ per minute until voluntary termination by the child despite strong verbal encouragement. HR and RPE were assessed throughout the exercise test. Peak work rate was based on a work rate value that was prorated based on the length of time for the final test stage (Bar-Or, 1983). Children with ADHD performed this test on medication as a previous study indicated medication use may affect peak exercise capacity (Mahon, Stephens, \& Cole, 2008).

The experimental conditions were carried out during the third and fourth visits to the laboratory. The procedures required the child to perform the CCPT II immediately prior to exercise, undertake a 20 -minute intermittent, high-intensity exercise protocol on the cycle ergometer followed immediately thereafter with another CCPT II. The 20-minute exercise bout was preceded by a 3-minute warm-up after which the child performed 30 seconds of exercise at $90 \%$ of peak working capacity, followed by 30 seconds of stationary rest. This exercise protocol resulted in a total of 10 minutes of high intensity exercise. An intermittent protocol was selected to facilitate compliance with completing each trial. HR and RPE were assessed throughout the exercise bout. Children with ADHD were assigned to perform one trial after 18-24 hours off-medication and the other trial 1-2 hours after ingesting their usual dose of medication in the morning in a counterbalanced manner. Given the half-life time course for amphetamine and methylphenidate, an 18-24 hours washout period is sufficient for conducting a trial off medication (Bennett, et al., 1999). Similarly, for the trial conducted on medication, peak behavioral effects of amphetamine and methylphenidate occur within 2 hours of consumption (Bennett, et al., 1999). For each subject in both groups these trials were carried out at approximately the same time of day.

\subsection{Research Design}

This study employed a mixed (within and between) subject research design. Group served as the between subject factor whereas trial and time served as within subject factors. Assignment to group was based on ADHD diagnosis. Trial order (with and without medication) for the children with ADHD was counterbalanced. The time factor represents before and after exercise assessments.

\section{Results}

\subsection{Statistical Analysis}

The physical characteristics (age, height, and weight), standardized K-BIT, BASC, and peak exercise responses were compared between groups using an independent t-test. A 3-way (group by trial by time) MANOVA was 
used to examine CCPT II performances, specifically the t-scores for errors of omission, errors of commission, and reaction time. Univariate ANOVA and Bonferroni post-hoc tests were used to isolate specific differences that resulted from main and interaction effects from the MANOVA. Because the initial MANOVA analysis with all subjects indicated a violation of Box's test of equality of covariance matrices, one subject from the ADHD group was randomly deleted so that all analyses were based on groups with equal sample sizes. Thus, the sample size used for these comparisons was 21 children in each group. Comparing groups of equal sample size serves as a means to make the MANOVA test conditionally robust to this violation (Stevens, 2002). All data are presented as means \pm standard deviations. A P-value of $\leq 0.05$ defined statistical significance.

\subsection{Physical Characteristics and Medication Use}

The physical characteristics of the participants are shown in Table 1. Both groups were of a similar $(p>0.05)$ age, but the control group tended to be taller $(p=0.08)$ and was heavier $(p \leq 0.05)$ than the ADHD group. For the children with ADHD, the average medication use per day was $32.0 \pm 14.7 \mathrm{mg}$ or $0.86 \pm 0.35 \mathrm{mg} / \mathrm{kg}$. Time since ADHD diagnosis was $4.7 \pm 2.2$ years and time on medication was $4.5 \pm 2.1$ years. Twelve children with ADHD were using methylphenidate and 9 children were using amphetamine for treatment. As for medication release type, 5 children were using immediate release and 16 children were using extended release versions of the medication.

Table 1. Physical characteristics

\begin{tabular}{lcc}
\hline \multicolumn{1}{c}{ Variable } & ADHD & Control \\
\hline Age $(\mathrm{yrs})$ & $11.3 \pm 1.8$ & $11.6 \pm 1.9$ \\
$\mathrm{Ht}(\mathrm{cm})$ & $144.6 \pm 15.0$ & $152.1 \pm 12.0$ \\
$\mathrm{Wt}(\mathrm{kg})$ & $38.2 \pm 12.1$ & $48.6 \pm 15.3^{\mathrm{a}}$ \\
\hline
\end{tabular}

$\operatorname{ADHD}(\mathrm{n}=21) ;$ Control $(\mathrm{n}=21){ }^{\mathrm{a}}=\mathrm{P} \leq 0.05$

\subsection{Peak Exercise Responses}

Work rate, heart rate and RPE at peak exercise were $116.2 \pm 34.6 \mathrm{~W}, 200 \pm 11 \mathrm{bpm}$, and $9.6 \pm 1.2$, respectively in the children with ADHD. In the control group these measurements were $129.4 \pm 31.4 \mathrm{~W}, 202 \pm 9 \mathrm{bpm}$, and $9.8 \pm$ 0.8 . There were no differences between groups for any of these variables.

\subsection{BASC and $K-B I T$}

The behavior (BASC) and intelligence scores for each group of children are listed in Table 2. There were no differences between groups with respect to the K-BIT score. At present, both the BASC hyperactivity and attention scores were higher $(\mathrm{p} \leq 0.05)$ in the children with ADHD child than in the control group. Also shown in Table 2 are the retrospective BASC scores for hyperactivity and attention in the children with ADHD.

Table 2. Behavioral and intelligence test scores

\begin{tabular}{lcc}
\hline \multicolumn{1}{c}{ Variable } & ADHD & Control \\
\hline K-BIT Score & $106.4 \pm 15.6$ & $107.7 \pm 13.4$ \\
Present BASC Hyperactivity & $58.5 \pm 15.6$ & $44.2 \pm 6.8^{\mathrm{a}}$ \\
Present BASC Attention & $62.1 \pm 8.0$ & $45.6 \pm 8.2^{\mathrm{a}}$ \\
Past BASC Hyperactivity & $75.6 \pm 14.9$ & \\
Past BASC Attention & $74.7 \pm 11.3$ & \\
\hline
\end{tabular}

$\operatorname{ADHD}(\mathrm{n}=21) ;$ Control $(\mathrm{n}=21) ;{ }^{\mathrm{a}}=\mathrm{P} \leq 0.05$

\subsection{Effect of Exercise on CCPT II Performance}

Performances on the CCPT II for both groups of children are shown in Table 3. Significant findings were noted for the group and time main effects, and the trial by group and time by group interactions. Specifically, reaction time t-score was higher in the ADHD group than in the control group and the error of omission t-score was 
higher in the post-exercise period compared to the pre-exercise measure. The group by trial interaction indicated the reaction time t-score was higher $(\mathrm{p} \leq 0.05)$ in the ADHD group in both trials versus the control group; the reaction time t-score also decreased $(\mathrm{p} \leq 0.05)$ from the first trial (off-medication) to the second trial (on-medication). The group by time interaction indicated the t-score for reaction time was higher $(\mathrm{p} \leq 0.05)$ in the ADHD group versus the control group both before and after exercise, this t-score also increased $(\mathrm{p} \leq 0.05)$ over time in the ADHD group, but not in the control group. There were no significant findings for the trial main effect and the trial by time and group by trial by time interactions.

Table 3. Performance on the CCPT II

\begin{tabular}{cccccc}
\hline \multirow{2}{*}{ CCPT II } & \multicolumn{2}{c}{ Trial 1 } & \multicolumn{2}{c}{ Trial 2 } \\
\hline Om & Group & Pre-Exercise & Post-Exercise & Pre-Exercise & Post-Exercise \\
& CON & $53.6 \pm 17.6$ & $55.6 \pm 13.9$ & $46.5 \pm 9.1$ & $51.3 \pm 14.4^{\mathrm{b}}$ \\
\multirow{2}{*}{ Cm } & ADHD & $47.6 \pm 4.1$ & $47.6 \pm 7.5$ & $47.0 \pm 7.6$ & $48.8 \pm 9.6$ \\
& CON & $51.5 \pm 10.7$ & $48.3 \pm 10.9$ & $45.4 \pm 12.3$ & $45.4 \pm 13.2$ \\
\multirow{2}{*}{ RT } & ADHD $^{\text {a }}$ & $50.9 \pm 11.6$ & $54.0 \pm 12.8$ & $44.8 \pm 8.8$ & $47.6 \pm 12.4^{\mathrm{c}, \mathrm{d}}$ \\
& CON $^{*}$ & $40.2 \pm 8.9$ & $38.3 \pm 9.7$ & $40.0 \pm 9.8$ & $40.1 \pm 10.6$ \\
\hline
\end{tabular}

ADHD ( $\mathrm{n}=21)$; Control $(\mathrm{n}=21)$; CCPT II variables expressed as t-scores; ${ }^{\mathrm{a}}=\mathrm{P} \leq 0.05$ group effect; $^{\mathrm{b}}=\mathrm{P} \leq 0.05$ time effect; ${ }^{\mathrm{c}}=\mathrm{P} \leq 0.05$ group-by-trial interaction; ${ }^{\mathrm{d}}=\mathrm{P} \leq 0.05$ group-by-time interaction; Trial $1=$ off medication for ADHD; Trial 2 = on medication for ADHD; $\mathrm{Om}=$ errors of omission; $\mathrm{Cm}=$ errors of commission; $\mathrm{RT}=$ reaction time

\section{Discussion}

This study examined the effect of an acute bout of aerobic exercise on indices of attention and impulsivity, as measured by the CCPT II, in a group of children with ADHD as well as in children without ADHD. Specifically, measurements of commission errors, omission errors, and reaction time were assessed. These measurements were obtained in children with ADHD in both the medicated and non-medicated states. It was hypothesized that in the non-medicated state exercise would improve performance on the CCPT II in children with ADHD so that there would be no difference between groups or with the effects observed on medication.

That the exercise stimulus used in the present study failed to improve performance on the CCPT II in children with ADHD when examined without medication does not support the study's hypothesis. However, this outcome is in accordance with results reported by Craft (1983) who found that exercise durations of 1-, 5- and 10-mnutes at a heart rate of $\sim 170 \mathrm{bpm}$ had no effect on cognitive performance on the WISC-R (Digit Span and Coding B) and the Illinois Test of Psycholinguistic Abilities in hyperactive boys compared to a no exercise trial. In contrast, there are a number of studies reporting positive effects of a single bout of exercise on neurocognitive function in children with ADHD (Chang, et al., 2012; Medina, et al., 2010; Pontifex, et al., 2013; Tantillo, et al., 2002). For example, Tantillo et al. (2002) reported that motor impersistence score improved following a graded exercise to maximum in boys, but not girls with ADHD or in a control group. Studies by Chang et al. (2012), Medina et al. (2010), and Pontifex et al. (2013) also reported acute exercise improved behavior-based assessments in children with ADHD. The study by Medina et al. is particularly interesting because they employed an exercise bout nearly identical to the exercise bout used in the present study and showed improved performance on 6 of 13 measurements from the CCPT II in comparison to a control (stretching exercise) condition. Studies by Chang et al. and Pontifex et al. used a continuous exercise protocol on a treadmill. In the Chang et al. study, children with ADHD exercised for 30 minutes (20 minutes at HR of $\sim 150 \mathrm{bpm}$ ). Following exercise performance on the Stroop Test and the Wisconsin Card Sorting Test was improved; no improvement was observed in a non-exercising group of children with ADHD. Pontifex et al. had children exercise on a treadmill for 20 minutes at $65-75 \%$ of maximal heart rate. Following exercise, children with ADHD showed improvement on response accuracy and post-error slowing of reaction time on an inhibitory control task (modified Eriksen flanker test). A control group of children without ADHD also showed pre- to post-exercise improvement in response accuracy, but not post-error slowing of reaction time. In the studies by Medina et al., Pontifex et al., and Tantillo et al. the results were observed in the absence of medication use on the day of testing. It is not entirely clear whether acute 
use of medication on the day of testing influenced the results in the study by Chang et al.

In order for exercise to be considered as an effective component in the overall treatment and management plan for individuals with ADHD, it is necessary to understand the characteristics of the exercise dose and how these characteristics are linked to positive outcomes. In regard to the dose of a single bout of exercise needed to elicit a positive effect both the duration and the intensity of exercise performed need to be considered. With respect to duration, there may be a threshold below which positive effects are not likely. The present study and the study by Craft (1983) employed exercise protocols that did not exceed 10 minutes in duration and also failed to report a positive effect on neurocognitive function. In contrast, other studies (Chang, et al., 2012; Medina, et al., 2010; Pontifex, et al., 2013; Tantillo, et al., 2002) examining the effect of a single bout of exercise on neurocognitive function in children with ADHD, and reporting positive effects, utilized exercise protocols that lasted for longer durations ( 20 minutes). Likewise, the exercise intensity necessary to evoke a positive response needs to be considered. In the present study a high intensity exercise protocol was used ( $90 \%$ of peak aerobic work rate) whereas Craft employed a moderate exercise intensity $(\mathrm{HR}=\sim 170 \mathrm{bpm})$. Neither of these studies showed exercise improved neurocognitive function. However, other studies employing similar exercise intensities (Chang, et al., 2012; Medina, et al., 2010; Pontifex, et al., 2013; Tantillo, et al., 2002) as well as one which used a progressively increasing exercise intensity (Tantillo, et al., 2002) protocol all reported positive outcomes. Since the exercise intensity component does not seem to vary between studies showing no effects versus studies showing positive outcomes, it would appear that moderate to vigorous exercise intensity is necessary to evoke a positive effect on neurocognitive function, but only if the duration of exercise is adequate. It also is useful to consider whether any acute effect of exercise can be amplified with regularly performed exercise. In this regard, Veret and colleagues (2012) had children with ADHD participate in a 10-week structured exercise program (3 $\mathrm{d} / \mathrm{wk}, 45 \mathrm{~min} / \mathrm{d}, 77 \%$ of maximal heart rate). Some but not all the children were on medication during this study. Improvements in some aspects of behavior were noted by parents and teachers using the CBCL. Attention and time target pondering on the Sky Search component of the Test for Everyday Attention in Children also were improved following the exercise program. All of these improvements were in comparison to a control group of children with ADHD who did not participate in the exercise program. Collectively this information is supportive of a positive benefit of exercise on neurocognitive function in children with ADHD. However, as suggested by Pontifex (2013), more research is needed to establish the optimal exercise intensity and duration stimulus that positively impacts behavior, neurocognitive function, and academic performance in children with ADHD as are carefully controlled studies examining the outcomes associated with regularly performed exercise (Berwid \& Halperin, 2012).

The mechanism by which exercise improves neurocognitive function in individuals with ADHD is unclear, but there are a number of possible means by which improvement will occur. From a neurochemical standpoint, aerobic exercise has been shown to increase dopamine concentration the neostriatum and nucleus acumbens in rodents (Sutoo \& Akiyama, 1996); diminished dopaminergic activity in these areas of the brain are directly involved with ADHD (Biederman, 2005; Biederman \& Faraone, 2005; Sagvolden \& Sergeant, 1998). In a study involving children with ADHD, Tantillo et al. (2002) examined the acute effect of aerobic exercise on several noninvasive indices of dopaminergic activity in the brain including spontaneous eye blink rate, acoustic eye blink startle response, and acoustic eye blink startle response latency. From pre- to post-exercise, eye blink rate increased in boys with ADHD, acoustic startle eye blink response amplitude increased in girls with ADHD, and acoustic startle eye blink response latency decreased in both boys and girls with ADHD. All these responses are consistent with increased dopaminergic activity in the brain. No changes were observed in any of these responses in children without ADHD. Additionally, Pontifex et al. (2013) examined event related potentials associated with the regulation of executive control from electroencephalogram recordings and observed improvements in some of these indices in children with ADHD following exercise. More intriguing is the consideration as to whether regularly performed exercise can induce long-term structural and functional changes in brain activity that attenuate behavioral problems associated with ADHD (Berwid \& Halperin, 2012).

Although the hypothesis of the study was not supported by the results, there are nonetheless several significant findings that warrant discussion. A group effect was noted with respect to reaction time. In particular reaction time performance was lower in the ADHD group compared to the control group. This finding is consistent with the nature of the disorder and the effect it has on vigilance and concentration (Sagvolden \& Sergeant, 1998). There also was a time effect that indicated exercise might actually worsen performance on the CCPT II as indicated by a significant increase in the error of omission t-score from pre- to post-exercise. The group-by-trial and group-by-time interaction effects also were significant for reaction time. The group-by-trial effect indicates that the performance on this aspect of the CCPT II improved in the ADHD group from the non-medication trial 
to the medication trial; an outcome consistent with the known effects of stimulant medication. The group-by-time interaction effect indicates that reaction time worsened slightly, but significantly, in the ADHD from pre-post-exercise and is consistent with the time main effect noted above.

\subsection{Limitations}

There are some limitations in the current study, which should be acknowledged. First, there were variations in the medication type and dose amount used by the children in this study. It is recognized that ideally these two factors would have been constant; however, a similar variation in medication use is apparent in recent studies that examined stimulant medication effects on exercise responses (Mahon, et al., 2008; Mahon, et al., 2012). Second, although approximately equal numbers of males and females comprised the control group, there were only two females in the group with ADHD. However, given that ADHD is diagnosed at a much higher frequency in boys than in girls it is not surprising this is the case (Benner-Davis \& Heaton, 2007; Castle, Aubert, Verbrugge, Khalid, \& Epstein, 2007; Gershon, 2002). Third, there was variation among the children with ADHD with regard to ADHD sub-type classification. Based on parental report 5 children were inattentive sub-type, 5 children were hyperactive/impulsive sub-type, 8 children were combined sub-type, and 3 parents were unable to classify their child's ADHD sub-type. It is not certain whether the variation among the children with respect to ADHD sub-type impacted the outcome of the study. Another limitation of this study is that several children had difficulty completing the 20-minute exercise bout. Specifically, 4 children with ADHD and 1 child without ADHD terminated the trial prior to completing all 20 of the 30 -second exercise intervals. However, all these children completed 16 to 19 of the 20 intervals and this was deemed sufficient for inclusion in the analyses and it is unlikely this had a negative impact on the results. Lastly, because the children were using their prescribed medication and majority were using extended release capsule version of their medication it was not practical to carry-out the experimental protocol in a blinded or dose-controlled manner.

\subsection{Summary}

In summary this study examined the effect of an acute bout of exercise on CCPT II performance in children with ADHD. It was anticipated that exercise in the non-medicated state would improve reaction time and measure of errors of omission and errors of commission in children with ADHD compared to a control group and a trial performed on medication. However, this was not case and may have been due to an insufficient stimulus with respect to exercise duration. Identifying the optimal exercise stimulus and conditions that improve cognitive and behavioral function in this population is warranted.

\section{Acknowledgement}

This research was supported with funding from the National Institute of Child Health and Human Development (grant \# 1-R03 HD042196-01A1). The authors also would like to acknowledge their appreciation to James A. Jones, Ph.D. for his advice on the statistical analysis.

\section{References}

AAP. (2001). American Academy of Pediatrics - Clinical practice guideline: Treatment of the school-aged child with attention-deficit/hyperactivity disorder. Pediatrics, 108, 1033-1044.

Achenbach, T. M. (1991). Integrative Guide to the 1991 CBCL/4-18, YSR, and TRF Profiles. Burlington, VT: University of Vermont.

APA. (2000). Diagnostic and Statistic Manual of Mental Disorders IV-TR. Washington, DC: American Psychiatric Association.

Bar-Or, O. (1983). Pediatric Sports Medicine for the Practitioner. New York: Springer-Verlag.

Barkley, R. A. (1998). Attention-deficit hyperactivity disorder. Scientific American, 279, 66-71.

Bass, C. K. (1985). Running can modify classroom behavior. Journal of Learning Disabilities, 18, 160-161.

Benner-Davis, S., \& Heaton, P. C. (2007). Attention deficit and hyperactivity disorder: Controversies of diagnosis and safety of pharmacological and nonpharmacological treatment. Current Drug Safety, 2, 33-42.

Bennett, F. C., Brown, R. T., Craver, J., \& Anderson, D. (1999). Stimulant medication for the child with attention-deficit/hyperactivity disorder. Pediatric Clinics of North America, 46, 929-944.

Berwid, O. G., \& Halperin, J. M. (2012). Emerging support for a role of exercise in attention-deficit/hyperactivity disorder intervention planning. Current Psychiatry Reports, 14, 543-551. http://dx.doi.org/10.1007/s11920-012-0297-4

Biederman, J. (2005). Attention-deficit/hyperactivity disorder: A selective overview. Biological Psychiatry, 57, 
1215-1220. http://dx.doi.org/10.1016/j.biopsych.2004.10.020

Biederman, J., \& Faraone, S. V. (2005). Attention-deficit hyperactivity disorder. Lancet, 366, 237-248. http://dx.doi.org/10.1016/S0140-6736(05)66915-2

Castle, L., Aubert, R. E., Verbrugge, R. R., Khalid, M., \& Epstein, R. S. (2007). Trends in medication treatment for ADHD. Journal of Attention Disorders, 10, 335-342. http://dx.doi.org/10.1177/1087054707299597

Chang, Y. K., Liu, S., Yu, H. H., \& Lee, Y. H. (2012). Effect of acute exercise on executive function in children with attention deficit hyperactivity disorder. Archives of Clinical Neuropsychology, 27, 225-237. http://dx.doi.org/10.1093/arclin/acr 094

Charach, A., Skyba, A., Cook, L., \& Antle, B. J. (2006). Using stimulant medication for children with ADHD: What do parents say? A brief report. Journal of the Canadian Academy of Child and Adolescent Psychiatry, $15,75-83$.

Connors, C. K., \& Staff, M. (2002). Connors' Continuous Performance Test II Computer Program for WindowsTechnical Guide and Software Manual. Toronto: Multi-Health Systems, Inc.

Craft, D. H. (1983). Effect of prior exercise on cognitive performance tasks by hyperactive and normal young boys. Perceptual and Motor Skills, 56, 979-982.

Gershon, J. (2002). A meta-analytic review of gender differences in ADHD. Journal of Attention Disorders, 5 , 143-154.

Greenhill, L. L. (1998). Diagnosing attention-deficit/hyperactivity disorder in children. The Journal of Clinical Psychiatry, 59 Suppl 7, 31-41.

Hale, J. B., Reddy, L. A., Decker, S. L., Thompson, R., Henzel, J., Teodori, A., ... Denckla, M. B. (2009). Development and validation of an attention-deficit/hyperactivity disorder (ADHD) executive function and behavior rating screening battery. Journal of Clinical and Experimental Neuropsychology, 31, 897-912. http://dx.doi.org/10.1080/13803390802687423

Higdon, H. (1999, July). Getting their attention. Runners' World, 84-87.

Kaufman, A. S., \& Kaufman, N. L. (1990). Chapter 5: Technical data: Reliability, intercorrelations, and validity. In Kauffman Brief Intelligence Test Manual (pp. 55-68). Circule Pines, MN: Pearson, Inc.

Kaufman, A. S., \& Kaufman, N. L. (2004). Kauffman Brief Intelligence Test (2nd ed.). Bloomington, MN: Pearson, Inc.

Mahon, A. D., Stephens, B. R., \& Cole, A. S. (2008). Exercise responses in boys with attention deficit/hyperactivity disorder: Effects of stimulant medication. Journal of Attention Disorders, 12, 170-176. http://dx.doi.org/ 10.1177/1087054707308484

Mahon, A. D., Woodruff, M. E., Horn, M. P., Marjerrison, A. D., \& Cole, A. S. (2012). The effect of stimulant medication use by children with attention deficithyperactivity disorder on the relationship between perceived exertion and heart rate. Adapted Physical Activity Quarterly, 29, 151-160.

Medina, J. A., Netto, T. L., Muszkat, M., Medina, A. C., Botter, D., Orbetelli, R., .. Miranda, M. C. (2010). Exercise impact on sustained attention of ADHD children, methylphenidate effects. Attention Deficit and Hyperactivity Disorders, 2, 49-58. http://dx.doi.org/10.1007/s12402-009-0018-y

Pontifex, M. B., Saliba, B. J., Raine, L. B., Picchietti, D. L., \& Hillman, C. H. (2013). Exercise improves behavioral, neurocognitive, and scholastic performance in children with attention-deficit/hyperactivity disorder. The Journal of Pediatrics, 162, 543-551. http://dx.doi.org/10.1016/j.jpeds.2012.08.036

Reynolds, C. R., \& Kamphaus, R. W. (1998). Behavior Assessment for Childen. Circle Pines, MN: AGS, Inc.

Robertson, R. J., Goss, F. L., Boer, N. F., Peoples, J. A., Foreman, A. J., Dabayebeh, I. M., ... Thompkins, T. (2000). Children's OMNI scale of perceived exertion: mixed gender and race validation. Medicine and Science in Sports and Exercise, 32, 452-458.

Sagvolden, T., \& Sergeant, J. A. (1998). Attention deficit/hyperactivity disorder--from brain dysfunctions to behaviour. Behavioural Brain Research, 94, 1-10. http://dx.doi.org/10.1016/S0166-4328(97)00164-2

Stevens, J. (2002). Applied Multivariate Statistics for Social Sciences. Mahwah, NJ: Lawrence Erlbaum Associates.

Sutoo, D. E., \& Akiyama, K. (1996). The mechanism by which exercise modifies brain function. Physiology \& 
Behavior, 60, 177-181.

Swanson, J. M., \& Volkow, N. D. (2009). Psychopharmacology: Concepts and opinions about the use of stimulant medications. Journal of Child Psychology and Psychiatry, and Allied Disciplines, 50, 180-193. http://dx.doi.org/10.1111/j.1469-7610.2008.02062.x

Tanner, J. M. (1962). Growth at Adolescence. Oxford: Blackwell Scientific Publishers.

Tantillo, M., Kesick, C. M., Hynd, G. W., \& Dishman, R. K. (2002). The effects of exercise on children with attention-deficit hyperactivity disorder. Medicine and Science in Sports and Exercise, 34, 203-212.

Verret, C., Guay, M. C., Berthiaume, C., Gardiner, P., \& Beliveau, L. (2012). A physical activity program improves behavior and cognitive functions in children with ADHD: an exploratory study. Journal of Attention Disorders, 16, 71-80. http://dx.doi.org/10.1177/1087054710379735

Volkow, N. D., Wang, G. J., Fowler, J. S., Gatley, S. J., Logan, J., Ding, Y. S., ... Pappas, N. (1998). Dopamine transporter occupancies in the human brain induced by therapeutic doses of oral methylphenidate. The American Journal of Psychiatry, 155, 1325-1331.

Wilens, T. E. (2006). Mechanism of action of agents used in attention-deficit/hyperactivity disorder. The Journal of Clinical Psychiatry, 67(Suppl 8), 32-38.

Wolraich, M. L. (1997). Addressing behavior problems among school-aged children: Traditional and controversial approaches. Pediatrics in Review / American Academy of Pediatrics, 18, 266-270.

Wolraich, M. L., McGuinn, L., \& Doffing, M. (2007). Treatment of attention deficit hyperactivity disorder in children and adolescents: safety considerations. Drug Safety: An International Journal of Medical Toxicology and Drug Experience, 30, 17-26.

\section{Copyrights}

Copyright for this article is retained by the author(s), with first publication rights granted to the journal.

This is an open-access article distributed under the terms and conditions of the Creative Commons Attribution license (http://creativecommons.org/licenses/by/3.0/). 